\title{
Género en la formación universitaria: re-tejiendo el hilo violeta en la UNLP
}

\section{Gender in university education: re-weaving the violet thread at National University of La Plata}

\section{Luisina Bolla}

http:/orcid.org/0000-0001-5860-8850

luisinabolla@gmail.com

Universidad Nacional de La Plata | CONICET Instituto de Investigaciones en Humanidades y Ciencias. Sociales | Centro Interdisciplinario de Investigaciones en Género | Argentina

\author{
Milagros María Rocha \\ http:/orcid.org/0000-0002-5361-0211 \\ milagrosmrocha@gmail.com \\ Facultad de Humanidades y Ciencias \\ de la Educación | Universidad Nacional \\ de La Plata | Argentina
}

\section{RESUMEN}

Desde el año 2015, ligado al impacto de las marchas Ni Una Menos, al Paro Nacional y a los Paros Internacionales de Mujeres (8 M), los reclamos de los movimientos feministas y de mujeres cobraron visibilidad en la agenda pública. El campo académico no permaneció al margen de estas interpelaciones y, en particular, las universidades comenzaron a fortalecer la inclusión de las perspectivas de género en el currículum y en sus diseños, por ejemplo, a través de la elaboración de protocolos y espacios institucionales específicos. El objetivo de este trabajo es continuar la senda de análisis iniciada en investigaciones previas (Rocha, 2019; Bolla y Rocha, 2020) para examinar de qué forma ingresan y se fortalecen los estudios feministas y de género en las universidades. En esta oportunidad abordamos dos ejes: enseñanza e investigación. Para seguir el recorrido del hilo violeta y sus conexiones (Ciriza y Rodríguez Agüero, 2021) nos preguntamos cuándo y de qué modo se incorporan dichas perspectivas o áreas en las universidades, intentando dar cuenta de los avances así como también de los desafíos que de allí se derivan. Para ello, retomamos algunos documentos e informes elaborados recientemente por redes y secretarías cuya creación es posterior al año $2015^{2}$. Luego, nos centramos en el caso específico de la UNLP, señalando antecedentes previos al año 2015, transformaciones y experiencias recientes en el abordaje e institucionalización de las temáticas feministas y de género.

2 Sobre el uso de los términos "feminismos" y "teoría de género" en este trabajo, ver Bolla y Rocha (2020).

PALABRAS CLAVE Universidad, género, enseñanza, investigación. 
KEY WORDS

University, gender,

teaching,

research.

\section{ABSTRACT}

Since 2015, linked to the impact of the Ni Una Menos demonstration, the National Strike and the International Women's Strikes (8 M), the demands of the feminist and women's movements gained visibility on the Argentinian public agenda. The academic field did not remain on the sidelines of these interpellations and, in particular, the universities began to strengthen the inclusion of gender perspectives in the curriculum and also through the development of protocols and specific institutional spaces. This paper continues the path of analysis started in previous research (Rocha, 2019; Bolla and Rocha, 2020) to examine how feminist and gender studies enter and are strengthened in universities. This time we address two axes: teaching and research. To follow the path of the violet thread and its connections (Ciriza and Rodríguez Agüero, 2021) we ask ourselves when and in what way these perspectives or areas are incorporated in the universities, trying to account for the progress as well as the challenges arised. In the first place, we take up some documents and reports recently prepared by networks and secretariats created after the year 2015. Then, we focus on the specific case of National University of La Plata (Argentina), pointing out antecedents prior to 2015, transformations and recent experiences in the approach and institutionalization of the feminist and gender themes. 


\section{ANTECEDENTES: UN ESTUDIO PRELIMINAR (DEL PRESENTE AL PASADO RECIENTE)}

De forma creciente desde el año 2014, las Universidades comenzaron a visibilizar y a trabajar en pos de la elaboración de los protocolos de actuación ante situaciones de discriminación y/o violencia de género. Según indica la Red interuniversitaria por la Igualdad de Género y contra las violencias (RUGE) en un informe de diciembre 2019, para esa fecha el $73,77 \%$ de las Universidades contaban con estos protocolos, $19,67 \%$ se encontraba en proceso y el $6,56 \%$ no tenía ${ }^{2}$. La Universidad Nacional del Comahue dio el puntapié inicial, continuando en 2015 las Universidades Nacionales de Córdoba, La Plata, San Martín y Buenos Aires. En dicho informe se indica que en el año 2014 se aprueba 1 protocolo; en 2015, 4; en 2016, 8; en 2017, 13 protocolos; en 2018, 11 y en 2019,8 , que hace un total de 45 Universidades con protocolo. El número creciente de Universidades con protocolo hace evidente el ingreso de esta problemática y la forma en que la visibilizaron y alojaron.

Las transformaciones que observamos en el sistema universitario de nuestro país se vinculan, en parte, con la creación en el año 2015 de la Red Interuniversitaria por la Igualdad de Género y contra las Violencias, con la participación de más de veinte universidades, facultades e institutos. En 2018, la Red se incorpora como organización en el marco del Consejo Interuniversitario Nacional (CIN), lo que contribuyó a profundizar el diseño y el desarrollo de políticas de género en el sistema universitario. La Red universitaria de género (RUGE) se focalizó en la creación y fortalecimiento de los protocolos de atención en situaciones de violencia de género y/o discriminación sexual, además de realizar relevamientos respecto al desarrollo de las políticas de género. Por

2 Fuente: DGyDS UNSAM y Coordinación Ejecutiva RUGE, 2019. Los datos que se dan a conocer se construyen en función de un relevamiento que "se realizó durante los meses de septiembre, octubre y noviembre del 2019. Dicho estudio de carácter cuantitativo relevó datos de 61 encuestas auto administradas realizadas a representantes de la Red Interuniversitaria por la Igualdad de Género y Contra las Violencias (RUGE) del Consejo Interuniversitario Nacional (CIN). El CIN está compuesto por un total de 66 instituciones universitarias, 57 nacionales, 4 provinciales y 5 institutos universitarios. El estudio relevó información de 61 instituciones universitarias: 56 universidades nacionales, 4 provinciales y el Instituto Patagónico de las Artes; dejando afuera 4 institutos universitarios y la Universidad Nacional Scalabrini Ortiz por encontrarse en proceso de normalización". Recuperada de: http://ruge.cin.edu.ar/attachments/ article/43/RUGE.\%20Relevamiento\%20principales\%20resultados\%200702\%20(4).pdf 
otra parte, en 2020, RUGE-CIN firma un convenio con la Iniciativa Spotlight, a través de ONU Mujeres. Dicha iniciativa es una alianza entre la Unión Europea y Naciones Unidas, buscando fortalecer las políticas de prevención y atención de la violencia de género en el sistema universitario, proponiendo una articulación entre Estado, sistema universitario y sociedad civil.

En continuidad con tal trabajo, a partir de 2019, muchas universidades adhieren a la "Ley Micaela3". Dicha ley hace explícita la necesidad de una capacitación obligatoria en la temática de género y violencia contra las mujeres, para todas las personas que ocupan cargos en la función pública de nuestro país, en todos sus niveles y jerarquías, en los poderes Ejecutivo, Legislativo y Judicial de la Nación. Luego de sancionarse la Ley, las Universidades comenzaron a adherir y a desarrollar dichas capacitaciones. Tiempo después, es el propio Consejo Interuniversitario Nacional (CIN) el que decide (por unanimidad) adherir a la Ley Micaela, haciendo de esta manera extensivo el alcance de la misma a todas las universidades públicas del país. En el marco de la ley, la formación está dirigida a docentes, estudiantes y no docentes.

Actualmente, los avances de la perspectiva de género son notorios y en buena hora derivaron en diversos cambios, acciones, leyes y normativas por parte del Estado y la Universidad como agente. Sin embargo, resulta necesario considerar los antecedentes previos al año 2015 que han sido un sustrato fundamental en el cual estas transformaciones recientes se siembran y crecen. Un trabajo de largo aliento que involucra los esfuerzos de diferentes docentes investigadorxs y activistxs tal como intentaremos recuperar a continuación.

En efecto, la inserción de las teorías feministas y de género en las universidades se abre camino desde los comienzos de la recuperación democrática, reconectando así con una larga y fecunda genealogía que, en nuestro país, se remonta a los comienzos del siglo XX, con la organización pionera de las primeras universitarias argentinas (Barrancos, 2002; Lobato, 2002, 2013; Spadaro, 2002)-aunque a los fines del presente trabajo circunscribimos nuestro análisis de la década del '80 en adelante-. La apertura de centros, institutos y/o posgrados que inscribieron las temáticas feministas y de género en la trama institucional sustentaron así la vía en la cual se amplifican los reclamos post-2015. Nos preguntamos entonces: ¿cómo se institucionaliza y se consolida la perspectiva de "género" o los estudios feministas en las universidades de nuestro país, desde el retorno de la democracia y posteriormente? ¿De qué forma comenzaron a abrirse los caminos que permitieron, en complejos procesos no siempre lineales, la creciente visibilización que el género ha ganado en las últimas décadas, dentro del ámbito universitario? 
la inserción de las teorías feministas y de género en las universidades se abre camino desde los comienzos de la recuperación democrática, reconectando así con una larga y fecunda genealogía que, en nuestro país, se remonta a los comienzos del siglo XX, con la organización pionera de las primeras universitarias argentinas (Barrancos, 2002; Lobato, 2002, 2013; Spadaro, 2002)-aunque a los fines del presente trabajo circunscribimos nuestro análisis de la década del ' 80 en adelante.

\section{GÉNERO, ENSEÑANZA E INVESTIGACIÓN EN LA UNIVERSIDAD NACIONAL DE LA PLATA}

Retomamos a continuación algunas líneas de investigación derivadas de trabajos individuales previos (Rocha, 2019) y conjuntos (Bolla y Rocha, 2020). Allí indagamos en la presencia/ausencia de mujeres en los programas de las materias correspondientes a la carrera de Historia (FaHCE-UNLP) y propusimos un análisis exploratorio sobre programas de Introducción a la Filosofía en algunas universidades nacionales. En ese contexto, propusimos la categoría de "currículum en disputa" (Bolla y Rocha, 2020) para dar cuenta de las disputas en torno a la incorporación de temas y autoras vinculadas a género y feminismos. Mostramos a su vez que dicha trama involucró a diferentes sujetos del espacio universitario, desde el claustro estudiantil hasta docentes de diferentes cátedras.

Para profundizar esos análisis, proseguimos ahora el abordaje de dos ejes: enseñanza e investigación, quedando pendiente la indagación de los desarrollos que tienen lugar en el área de la extensión universitaria. Tomamos como base las entrevistas y cuestionarios (de elaboración propia e institucionales) realizados a diferentes actores del ámbito universitario y el análisis de informes y programas de la UNLP, en diálogo con la bibliografía específica.

\section{GÉNERO Y ENSEÑANZA}

En el caso del eje enseñanza, pudimos complementar nuestras investigaciones previas con nuevos materiales elaborados en los últimos dos años. En 2019, la Dirección de Políticas Feministas de la Universidad Nacional de La Plata da a conocer un informe preliminar: "la perspectiva de género en la UNLP". Dicho informe lo elaboran en base a las respuestas recibidas de parte de diez Unidades Académicas sobre un total de diecisiete. Este documento puntualiza en las áreas de enseñanza, investigación, extensión, protocolo, entre otras. Respecto a la enseñanza, indica que el $60 \%$ de las facultades tiene materias donde se aborda la perspectiva de género, mientras que el $40 \%$ por 
el momento no posee. En cuanto a las materias específicas sobre género, indica que el $30 \%$ de las facultades relevadas cuenta con ellas, mientras que el $70 \%$ no. En cuanto a los contenidos mínimos, señala que el $40 \%$ incluyen la perspectiva de género, mientras que el $60 \%$ no lo contempla.

Si bien estas estadísticas aportan indicadores sumamente valiosos, también nos preguntamos por la visibilidad, más precisamente, por el lugar otorgado a estos temas en el currículum universitario. Siguiendo nuestras investigaciones previas, observamos que en algunas oportunidades estos temas y textos ingresan de manera excepcional, mínima y en otros como sección "especial". Nos preguntamos en ese sentido de qué modo la perspectiva interpela al canon, no sólo en términos cuantitativos ya sea de contenidos o textos, sino en términos generales: ¿en qué medida se logra interpelar lo instituido? ¿De qué forma se problematiza la "historia única" del currículum, retomando la sugerente metáfora de la escritora nigeriana Chimamanda Ngozi Adichie (2018 [2009])?

En el año 2018, una agrupación estudiantil de la Facultad de Humanidades y Ciencias de la Educación de la UNLP, exploró los porcentajes de autores y autoras presentes en las materias del primer año de las diversas carreras. Los resultados dados a conocer fueron: en las carreras de Historia y Filosofía, $87 \%$ varones, $13 \%$ mujeres; en Educación Física y Letras, 78\% varones, 22\% mujeres; en Traductorado de inglés, $74 \%$ varones y $26 \%$ mujeres; en Sociología, $83,4 \%$ varones, $16,6 \%$ mujeres. La contundencia de estos resultados nos motivó a seguir interpelando los espacios y las formas de construir el conocimiento y su enseñanza, así como las formas y prácticas sedimentadas que reproducen lógicas patriarcales. Recientemente, la agrupación volvió a realizar ese ejercicio y análisis, contemplando los programas actualizados de las materias de primero y segundo año. Y dio a conocer las estadísticas, publicadas en junio 2021: Educación Física, 86\% varones, 14\% mujeres; Traductorado de Inglés, 53\% varones, 47\% mujeres; Historia, 80,20\% varones, 19.80\% mujeres; Ciencias de la Educación, 55,80\% varones y 44,20\% mujeres; Sociología, $83,80 \%$ varones y $16,20 \%$ mujeres; Filosofía, $76,60 \%$ varones $23,40 \%$ mujeres; Letras, 74,40\% varones, 25,60\% mujeres; Geografía, 58,30\% varones y $41,70 \%$ mujeres; Bibliotecología, 52,30\% y 47,70\% mujeres. En las carreras de Traductorado, Ciencias de la Educación y Bibliotecología, aparece un porcentaje similar; la diferencia no es tan notoria como en otras carreras, como es el caso de Educación Física, Historia, Sociología, Filosofía y Letras.

Retomando a Walter Benjamin, el currículum hegemónico ha sido elaborado por sujetos igualmente hegemónicos que instauran una genealogía basada en lo que Charles Taylor (1992), no sin provocación, denominó los "varones blancos muertos". En filosofía, por ejem- 
plo, los debates que los filósofos mantenían en su época, las tesis con las que se peleaban y discutían, y en este caso en particular, las interlocuciones con filósofas y filósofos que cuestionaban modelos epistémicos basados en la exclusión, rara vez son referidos. Se les resta autoridad epistémica y se los excluye del ámbito del saber considerado valioso y legítimo (Maffía, 2020).

Sin embargo, estas exclusiones han sido históricamente objeto de luchas y disputas. En el informe de la Dirección de Políticas Feministas ya mencionado, se detallan los años a partir de los cuales se dictan materias específicas sobre género en distintas unidades académicas (Cuadro 3: "Año en que empiezan a dictarse materias sobre género"). La Facultad de Humanidades y Ciencias de la Educación sería una pionera al contar con materias específicas desde el año 2008; seguida por Ciencias Naturales (2014), Trabajo Social (2015), Odontología (2016), hasta las experiencias más recientes de Derecho y Económicas (2019).

En el caso de la Facultad de Humanidades, se pueden referenciar antecedentes previos. Por caso, en las carreras de Historia y de Filosofía, encontramos materias optativas que incorporan en su enfoque las perspectivas feministas y de género. En Filosofía, desde el año 2001, la materia "Antropología Filosófica" incorpora de manera creciente una mirada crítica basada en los aportes que realizan los estudios de género (Femenías, 2002) ${ }^{4}$. Como señala María Luisa Femenías, quien fuera titular hasta el año 2015:

comencé a dar Antropología Filosófica que no se daba como hacía 15 años y se la reflotó, porque en algún momento se daba una antropología que era una teología antropológica, los chicos no la querían leer, y armamos un programa muy diferente, introduciendo otras cosas, otras corrientes y ahí comenzó a tener un perfil un poco más interesante para mi gusto (Femenías en Martínez y Bolla, 2021, s/p).

En la actualidad, la materia -a cargo de Mabel A. Campagnoli, sub-directora del CInIG- continúa desarrollando un enfoque crítico que hilvana teorías feministas y de género con aportes de las corrientes pos y decoloniales 5 .

En otras carreras, como Historia, también encontramos antecedentes relevantes. El Seminario/Materia Problema a cargo de Adriana Valobra -que también se desempeña como directora del CInIG-, dicta, desde 2005 y de manera continua, esta materia que va cambiando sus títulos y contenidos pero que mantiene estos temas como ejes centra-

\footnotetext{
4 Pueden consultarse los programas en:https://memoria.fahce.unlp.edu.ar/programas/pp.5568/ pp.5568.pdfhttps://www.memoria.fahce.unlp.edu.ar/programas/pp.543/pp.543.pdfhttps://www.memoria.fahce.unlp.edu.ar/programas/pp.6766/pp.6766.pdf

5 https://www.memoria.fahce.unlp.edu.ar/programas/pp.10772/pp.10772.pdf
} 
les e incorpora en 2017 "las masculinidades a debate" ${ }^{6 "}$ Asimismo hallamos otros antecedentes: en 1989, Silvia Mallo da una materia denominada "La mujer en el período colonial" Molas, dicta la materia "Mujer, familia y sexualidades en la Argentina en los siglos XVII al XX"."

A su vez mencionamos que en 2016 la Facultad crea una materia optativa dirigida a todas las carreras de la Unidad Académica, dependiente de Secretaría Académica, denominada "Introducción a la teoría feminista y de género".

Por último, referenciamos que desde 2018 la Universidad organiza los talleres de género en los ingresos con la finalidad de visibilizar la perspectiva, avanzando, recientemente, en pensar la transversalización en dichas instancias.

Observamos entonces cómo estos temas ingresan en grado en diferentes temporalidades. Cada Unidad Académica va tejiendo su memoria en relación al tema bajo estudio, con diversos recorridos y antecedentes. Esas particularidades permiten ir construyendo ese hilo violeta al interior de la Universidad. Para cerrar, pero abriendo la pregunta, nos preguntamos por qué estos contenidos han permeado con mayores desarrollos en espacios curriculares optativos.

\section{Observamos entonces cómo estos temas ingresan en grado en diferentes temporalidades. Cada Unidad Académica va tejien- do su memoria en relación al tema bajo estudio, con diversos recorridos y antecedentes. Esas particularidades permiten ir construyendo ese hilo violeta al interior de la Universidad.}

\section{GÉNERO E INVESTIGACIÓN}

Emprendemos ahora un breve recorrido por el eje investigación. En ese sentido, indagamos y sistematizamos el surgimiento de áreas, laboratorios o centros de las mujeres, feministas o de género en nuestro país, desde mediados de la década de 1980 en adelante. No sin obstáculos y trabas institucionales, las Áreas o Centros de la Mujer, muchos re-nombrados luego desde el "género", dieron inicio a una tradición de análisis en diferentes universidades.

\footnotetext{
$6 \quad$ Pueden consultarse los programas en: https://www.memoria.fahce.unlp.edu.ar/programas/ pp.102/pp.102.pdf,https://www.memoria.fahce.unlp.edu.ar/programas/pp.259/pp.259.pdf, https://www. memoria.fahce.unlp.edu.ar/programas/pp.6837/pp.6837.pdf,https://www.memoria.fahce.unlp.edu.ar/ programas/pp.8028/pp.8028.pdf, https://www.memoria.fahce.unlp.edu.ar/programas/pp.8376/pp.8376. pdf, https://www.memoria.fahce.unlp.edu.ar/programas/pp.10702/pp.10702.pdf

7 Se puede consultar el programa en: https://www.memoria.fahce.unlp.edu.ar/programas/ pp.4280/pp.4280.pdf

8 Se puede consultar el programa en: https://www.memoria.fahce.unlp.edu.ar/programas/ pp.5857/pp.5857.pdf
} 
Entre los antecedentes, se destaca en Rosario el Centro de Estudios Históricos Sobre las Mujeres (CEHM), radicado en la Escuela de Historia de la Facultad de Humanidades y Artes de la Universidad, impulsado principalmente por Hilda Habichayn. Este centro en 1991 cambia su denominación por Centro de Estudios Interdisciplinario sobre las Mujeres (CEIM, UNR), incorporando como su nombre indica un centro interdisciplinario. Allí se crea la primera Maestría en Latinoamérica sobre género, en abril de 1993, denominada "Maestría Interdisciplinaria sobre la Problemática del Género en Latinoamérica" más tarde denominada "Poder y Sociedad desde la Problemática de Género" (nombre no elegido sino impuesto luego de varios intercambios con CONEAU $^{9}$ ). En la década de los 90' se crea la Revista Zona Franca, una publicación que viene a reemplazar los Cuadernos de CEHM (primeras instancias de difusión de los trabajos del CEHM y actividades académicas), así como la creación de un Centro de Documentación de Género y Feminismo. Alejandra Ciriza y Eva Rodríguez Agüero (2021) dan cuenta de estos antecedentes, entre otros, construyendo una genealogía de los estudios feministas en las universidades nacionales. Este zurcido, en clave federal, permite avizorar las referencias institucionales que van dando lugar al tema. Ciriza y Rodríguez Agüero muestran cómo desde Rosario al NOA y desde Luján al Comahue, va surgiendo la idea de crear centros de investigación sobre género y feminismos y referencian puntos en común. Sin dudas, la visita de Reyna Pastor en 1989 en nuestro país, luego de su exilio, fue la llave que contribuyó a potenciar estos procesos. En esta red de vínculos institucionales destacamos la creación en 1996 de la Revista La Aljaba, segunda época, editada por las Universidades de La Pampa, Luján y Comahue, siendo Coeditoras María Herminia Di Liscia (UNLPam); Nélida Bonaccorsi (UNCo) y Cecilia Lagunas (UNLu).

Este zurcido, en clave federal, permite avizorar las referencias institucionales que van dando lugar al tema. Ciriza y Rodríguez Agưero muestran cómo desde Rosario al NOA y desde Luján al Comahue, va surgiendo la idea de crear centros de investigación sobre género y feminismos y referencian puntos en común. Sin dudas, la visita de Reyna Pastor en 1989 en nuestro país, luego de su exilio, fue la llave que contribuyó a potenciar estos procesos.

\footnotetext{
9 Hemos tomado como fuente para estos datos, el correo que envió el Instituto de Investigaciones de Estudios de Género (Facultad de Filosofía y Letras, UBA) firmado por su directora actual Mónica Tarducci, con fecha del 12 mayo 2021, tras la muerte de Hilda Habichayn, entre otras fuentes.
} 
En la Universidad de Buenos Aires, se destaca la creación del Instituto de Investigaciones en Estudios de Género (IIEGE) de la Facultad de Filosofía y Letras, en el año 1997; dirigido desde el año 2000 por la historiadora Dora Barrancos. El antecedente del IIEGE fue el Área Interdisciplinaria de Estudios de la Mujer (AIEM) fundada en julio de 1992. Es en la década de los '90 que lanza la Revista Mora.

En la Universidad Nacional de La Plata, en el año 2006 se creó el Centro Interdisciplinario de Investigaciones en Género (CInIG), aprobado formalmente en el año 2007 por la Secretaría de Ciencia y Técnica de la UNLP (res. no 49/07 del Consejo Superior). Su primer director fue el Dr. José Amícola y su co-directora la Dra. María Luisa Femenías. Actualmente dirigido por la Dra Adriana Valobra. Dicho Centro cuenta, desde 2017, con la Revista Descentrada.

\begin{abstract}
Para recapitular, en el caso de la UNLP, el primer centro de investigación específico sobre estas temáticas fue el ClnIG, impulso que sería continuado en años posteriores con la creación de Laboratorios, Áreas y Centros de Género en otras facultades (Periodismo y Comunicación Social, Trabajo Social, entre otras). De ese modo, comienza a hilvanarse el "hilo violeta" entre las diferentes unidades académicas de la UNLP, en un proceso que continúa a la fecha y que se acompaña de otras iniciativas a nivel institucional (creación de Secretarías y Prosecretarías, Direcciones de Políticas Feministas y de Género, Oficinas y Áreas con incumbencia específica para la atención e implementación de Protocolos contra la Violencia, etc.).
\end{abstract}

Para recapitular, en el caso de la UNLP, el primer centro de investigación específico sobre estas temáticas fue el ClnIG, impulso que sería continuado en años posteriores con la creación de Laboratorios, Áreas y Centros de Género en otras facultades (Periodismo y Comunicación Social, Trabajo Social, entre otras). De ese modo, comienza a hilvanarse el "hilo violeta" entre las diferentes unidades académicas de la UNLP, en un proceso que continúa a la fecha y que se acompaña de otras iniciativas a nivel institucional (creación de Secretarías y Prosecretarías, Direcciones de Políticas Feministas y de Género, Oficinas y Áreas con incumbencia específica para la atención e implementación de Protocolos contra la Violencia, etc.). Sin embargo, si miramos el caso puntual del área de investigación, observamos que el CInIG constituye un antecedente relativamente reciente en relación con el desarrollo de otros centros en Universidades Nacionales. Vinculado a ello, también es relativamente reciente la creación de su revista asociada (Descentrada). 
No obstante, existieron antecedentes tempranos en nuestra Universidad. Una primera experiencia transformadora fue la creación de la cátedra libre "de la Mujer", a cargo de Silvia Knight de Carriquiriborde, en los años 80, durante la presidencia de Raúl Alfonsín. Aunque hemos comenzado a indagar acerca de este espacio, consultando diversas fuentes y personas referentes del campo, a la fecha contamos con pocos datos que nos permitan ampliar este antecedente. Aún en ausencia de mayores informaciones, nos parece relevante mencionarlo dado que constituyó una experiencia pionera en nuestra Universidad que deberemos continuar investigando.

\section{REFLEXIONES FINALES}

A lo largo de estas páginas, hemos intentado "tirar" del hilo violeta para buscar sus tramas e inicios en la UNLP, desde la década de 1980 hasta la actualidad. Se trata, por supuesto, de una indagación exploratoria que abreva de los resultados parciales de análisis previos y que, a la vez, indica los caminos que deberán continuarse a fines de complejizar y enriquecer este panorama. Mostramos que durante la recuperación democrática y los años posteriores, tanto las voluntades individuales como los esfuerzos grupales o colectivos puntuales, le han dado visibilidad al tema y permitieron abrir un espacio epistémico y político fecundo. En la actualidad, observamos también un impulso institucional, más evidente y transversal, que marca un fuerte contraste con el pasado.

Por su parte, advertimos los antecedentes pioneros que visibilizan y legitiman estos temas en las áreas y centros de investigación, en las diversas universidades de nuestro país. Recordamos que muchos de ellos fueron creados durante los años ' 90 . No obstante, los importantes avances y los desarrollos generados tanto desde los Centros como desde los posgrados universitarios, no mantienen el mismo correlato al nivel de las carreras de grado, como sostienen Barrancos (2019), Martin (2019), Fabbri y Roveto (2020), Cruz (2021); hecho que por nuestra parte también afirmamos a la luz de los resultados de nuestras indagaciones.

Por otra parte, la desigualdad en términos de enseñanza e investigaciones sigue siendo persistente. Según indica el informe preliminar de la Dirección de Políticas Feministas de la Universidad Nacional de La Plata (2019) ya mencionado, sólo un $2 \%$ de los proyectos de investigación radicados en las unidades académicas que respondieron la encuesta analizan específicamente o incluyen el género como objeto de estudio. Otro tanto ocurre con una visibilidad de excepcionalidad o un formato de tema especial, muchas veces suele ser el modo en que se da lugar al género y a los feminismos en los programas universitarios. Esta problemática se refuerza al considerar los bajos porcentajes de 
autoras mujeres, aún a sabiendas de que ello tampoco implica una tematización específica sobre el género. Si las universidades continúan siendo territorios del patriarcado (Morgade, 2018), sólo mediante las acciones y tramas colectivas podremos desarmar la empeñosa rigidez tan lúcidamente identificada por Dora Barrancos (2019). En ese camino nos encontramos. 


\section{BIBLIOGRAFIA}

Adichie, C. N. (2018 [2009]). El peligro de la historia única. Barcelona: Random House.

Arce, I. y Moreno, M. M. (2015). C. E. I. M. Un espacio con memoria. En: Koldorf, A. E. (comp.). Generando conexiones: aportes desde el feminismo y el concepto de género al uso y acceso responsable de las TICs. Rosario. Del Revés.

Barrancos, D. (2019). Devenir feminista. Una trayectoria político-intelectua. Martín, A.L. y Valobra, A. (comps). - 1a ed. - Ciudad Autónoma de Buenos Aires: CLACSO; Ciudad Autónoma de Buenos Aires : Editorial de la Facultad de Filosofía y Letras.

Barrancos, D. (2002). Cien años de feminismo en la Argentina: Jornada de homenaje a Elvira López, cien años de estudios feministas en la Argentina. Mora, 8

Benjamin, W. (2001). Tesis de filosofía de la historia en Ensayos escogidos. México: Coyoacán.

Bolla, L., \& Rocha, M. M. (2020). Género y currículum en disputa. Reflexiones sobre prácticas y saberes universitarios. Revista Tempo E Argumento, 12(30), e0207. https://doi.org/10.5965/2175180312302020e0207

Castro Gómez, S. (2015). Decolonizar la universidad:la hybris del punto cero y el diálogo de saberes.In: PALERMO, Zulma. Des/colonizar la universidad. Buenos Aires:Del Signo.

Ciriza, A. y Rodríguez Agüero E. (2021). Genealogías de los estudios feministas en las universidades nacionales: una cartografía provisoria. En: Martin A. L. (comp.). RUGE,El género en las universidades. CIN-RUGE.

Cruz, V. (2021). La institucionalización de la perspectiva de género en universidades públicas: apuntes sobre experiencias (de)construidas y pendientes. En: Martin, A. L.(comp). RUGE, el género en las universidades. RUGE

Femenías, M. L. (2015). ¿Qué le dan el feminismo y la teoría de género a la filosofía y su enseñanza?. In: CERLETTI, Alejandro y COULÓ, Ana (orgs.) La enseñanza filosófica: cuestiones de política, género y educación.Buenos Aires: Noveduc,

Fabbri, L. y Rovetto, F. (2020) Introducción. Cuadernos feministas para la transversalización. En: Maffía, D. [et. al] Apuntes epistemológicos. Rosario: Editorial de la Universidad Nacional de Rosario. 
Femenías, M. L. (2009). Género y feminismo en América Latina. Debate Feminista, año 20, n. 40, p. 42-74.

Lobato, M. Z. (2002) El movimiento feminista y la situación de la mujer en las palabras de Elvira López: selección documental. Mora, 8.

Lobato, M. Z. (2013) Las rutas de las ideas: «cuestión social», feminismos y trabajo femenino. Revista de Indias, vol. LXXIII, nº 257.

Maffía, D. (2007). Epistemología feminista: la subversión semiótica de las mujeres en la ciencia. Revista venezolana de estudios de la mujer, v. 12, n. 28, p. 63-98, 2007.

Maffía, D. (2020). Apuntes epistemológicos. Rosario: UNR Editora.

Martín, A. L. (2019). Trazos de una trayectoria inmarcesible. Aportes de Dora Barrancos a la relación entre género y universidad y entre ciencia y derechos. En: Barrancos, D. Devenir feminista. Una trayectoria político-intelectual. Dora Barrancos; compilado por Ana Laura Martín ; Adriana María Valobra. - 1a ed. - Ciudad Autónoma de Buenos Aires: CLACSO ; Ciudad Autónoma de Buenos Aires: Editorial de la Facultad de Filosofía y Letras.

Martín, A. L. (comp.) RUGE, el género en las universidades. Recuperado de:https://utn.edu.ar/images/Secretarias/SAE/RUGE/RUGE-libro-digital_ El-gnero-en-las-Universidades.pdf

Martínez, A. y Bolla, L. (2021). Entrevista a María Luisa Femenías. En Viguera, A., Tau, R. \& Vadura, N. (Comps.). Testimonios. Para una Historia Oral de la Psicología en la Universidad Nacional de La Plata. La Plata: Edulp. (En proceso de edición).

Morgade, G. (2018). Las Universidades Públicas como territorios del patriarcado.

Política Universitaria: La universidad hoy, a 100 años de la Reforma. [Buenos Aires]: Instituto de Estudios y capacitación Federación Nacional de Docentes Universitarios, v. 1.

Rocha, M. (2019). Mujeres y currículum en la formación universitaria: acerca del profesorado en historia de la Universidad Nacional de La Plata (19932017). Tesis

(Maestría en Educación). Facultad de Humanidades y Ciencias de la Educación, Universidad Nacional de La Plata, 2019.

Rovetto F... [et al.] (2020). Ley Micaela en el sistema universitario nacional: propuesta pedagógica para la formación y sensibilización en género y sexualidades 
/ - 1a ed.- Ciudad Autónoma de Buenos Aires: RUGE-CIN.Libro digital,PDF

Rovetto, F. y Figueroa, N. (2017). -Que la universidad se pinte de feminismos para enfrentar las violencias sexistas. Descentrada, 1(2), e026. Disponible en: http://www.descentrada.fahce.unlp.edu.ar/article/view/DESe026

Spadaro, M. (2002). Elvira López y su tesis 'El movimiento feminista' (1901): educación de las mujeres, camino hacia una sociedad más justa. Mora, 8.

Taylor, C. (1992). El multiculturalismo y "la política del reconocimiento. México: Fondo de Cultura Económica.

\section{Documento}

Universidad Nacional de La Plata, Prosecretaría de Derechos Humanos, Informe preliminar "la perspectiva de género en la UNLP", 2019. 